\title{
Pharmacologic Management
}

National Cancer Institute

\section{Source}

National Cancer Institute. Pharmacologic Management. NCI Thesaurus. Code C21090.

A health/illness/pain management principle specifically directed at the management of pharmacologic agents in maximizing beneficial outcomes and minimizing unfavorable effects. 